\title{
FACIAL REDUCTION FOR A CONE-CONVEX PROGRAMMING PROBLEM
}

\author{
JON M. BORWEIN and HENRY WOLKOWICZ
}

(Received 21 March 1980; revised 28 July 1980)

Communicated by J. B. Miller

\begin{abstract}
In this paper we study the abstract convex program

$$
\mu=\inf \{p(x): g(x) \in-S, x \in \Omega\}
$$

where $S$ is an arbitrary convex cone in a finite dimensional space, $\Omega$ is a convex set and $p$ and $g$ are respectively convex and $S$-convex (on $\Omega$ ). We use the concept of a minimal cone for $(\mathrm{P})$ to correct and strengthen a previous characterization of optimality for (P), see Theorem 3.2. The results presented here are used in a sequel to provide a Lagrange multiplier theorem for (P) which holds without any constraint qualification.
\end{abstract}

1980 Mathematics subject classification (Amer. Math. Soc.): 90 C 25.

Keywords: convexity, abstract programming, facial reduction, exposed face.

\section{Introduction}

Stimulated by a recent paper of Massam (1979), we study abstract convex programs in which the constraint function has finite dimensional range. In section two we define our optimization problem (P) and present the necessary preliminaries. In section three we introduce the concept of a minimal cone for (P) and use this minimal cone to correct and strengthen Massam's results. Lemmas 3.1 and 3.2 given here, will be used in Borwein and Wolkowicz (1980) to derive a Lagrange multiplier theorem for (P), which holds without any constraint qualification.

Copyright Australian Mathematical Society 1981

This research was partially supported by NSERC A4493 and NSERC A3388. 


\section{Preliminaries}

In this paper we consider the convex programming problem

$$
\mu=\inf \{p(x): g(x) \in-S, x \in \Omega\},
$$

where $p: X \rightarrow \mathbf{R} \cup\{+\infty\}, g: X \rightarrow Y \cup\{+\infty\} ; X$ and $Y$ are real locally convex (separated topological vector) spaces; $Y$ is finite dimensional with an abstract maximal element $+\infty$ (Peressini (1967)); $\Omega \subset X$ and $S \subset Y$ are convex and moreover $S$ is a cone (not necessarily closed), that is $\lambda s$ lies in $S$ whenever $s$ is in $S$ and $\lambda$ is non-negative; $p$ is a convex (extended) functional and $g$ is $S$-convex (on $\Omega$ ), that is

$$
t g\left(x_{1}\right)+(1-t) g\left(x_{2}\right)-g\left(t x_{1}+(1-t) x_{2}\right) \in S,
$$

for any $x_{1}, x_{2}$ (in $\Omega$ ) and any $t$ in $[0,1]$.

The set of $x$ for which $g(x)$ is finite is the domain of $g$, dom $g$. From (2.2) it follows that dom $g$ is convex. As is well known a convex cone $S$ induces an ordering $\geqslant_{s}$ on $Y$ given by

$$
x_{1} \geqslant x_{2} \Leftrightarrow x_{1}-x_{2} \in S
$$

which is transitive and reflexive. It is antisymmetric exactly when $S$ is pointed, that is $S \cap-S=\{0\}$. It will be convenient to introduce the following notation

$$
F=g^{-1}(-S), \quad A=g^{-1}(-S) \cap \Omega .
$$

Thus $A$ is the feasible set for (P) and we make the additional routine assumption that

$$
\operatorname{dom} p \supset A \neq \varnothing \text {. }
$$

Let $X^{*}$ and $Y^{*}$ denote the continuous dual spaces of $X$ and $Y$ respectively. We suppose throughout that $X^{*}$ is endowed with the weak star topology $\sigma\left(X^{*}, X\right)$ (see Robertson and Robertson (1964) for details).

Given any set $K$ in $X$ the dual cone of $K$ is the set in $X^{*}$

$$
K^{+}=\left\{x^{\prime} \in X^{*}: x^{\prime} x \geqslant 0 \text {, if } x \in K\right\} .
$$

Correspondingly if $K^{\prime}$ is in $X^{*}$

$$
K^{\prime+}=\left\{x \in X: x^{\prime} x \geqslant 0 \text {, if } x^{\prime} \in K^{\prime}\right\} .
$$

Then $K^{+}\left(K^{\prime+}\right)$ is always a closed convex cone and

$$
K^{++}=\left(K^{+}\right)^{+}\left(\left(K^{+}\right)^{+}\right)=\overline{\operatorname{coco} K},
$$

where $\overline{\operatorname{coco} K}$ denotes the closure of the convex cone generated by $K$. In particular, for a convex cone $S, S^{++}=\bar{S}$. The functionals in $S^{+}$will be said to be positive (for $S$ ). Given any two convex cones $S_{1}$ and $S_{2}$ in $X$

$$
\left(\bar{S}_{1} \cap \bar{S}_{2}\right)^{+}=\overline{S_{1}^{+}+S_{2}^{+}} \text {. }
$$


For proofs of these and other related results the reader is refered to Borwein (1978) and Holmes (1975). It will also be convenient to denote the annihilator of a set $K$ in $X$

$$
K^{\perp}=K^{+} \cap\left(-K^{+}\right) .
$$

The directional derivative of $g$ at $a$ is defined by

$$
\nabla g(a ; d)=\lim _{t \downarrow 0} \frac{g(a+t d)-g(a)}{t} .
$$

Then $\nabla g(a ; d)$ will exist for each direction $d$ if $g$ is convex on $X$, continuous at $a$ and $S$ is closed and pointed, Zowe (1974).

A continuous linear operator $T: X \rightarrow Y$ is a subgradient for $g$ at $a$ if

$$
T d \leqslant_{s} g(a+d)-g(a) \text { for all } d \text { in } X \text {. }
$$

The set of all such subgradients is denoted $\partial g(a)$. In case $Y=\mathbf{R}, S=\mathbf{R}_{+}$these definitions reduce to the standard ones and so apply to $p$. It follows from a result of Zowe's (1974) and (1975) that when $X$ is a weakly compactly generated Banach space (for example $X$ is reflexive or separable), and $g$ is convex on $X$ and continuous at $a$, with $S$ closed pointed and convex, then $\partial g(a)$ is non-empty and for any $s^{+}$in $S^{+}$

$$
s^{+} \nabla g(a ; d)=\max _{T \in \mathrm{\partial} g(a)} s^{+} T(d),
$$

for each $d$ in $X$. When $Y=\mathbf{R}$ and $S=\mathbf{R}_{+}$, then (2.13) holds true in any locally convex space $X$. Note that when $\nabla g(a ; t d)$ exists, then for $t>0$

$$
g(a+t d)-g(a)-\nabla g(a ; t d) \in \bar{S} .
$$

The reader is refered to Zowe (1974) and Borwein (1980) for more details.

Any other terms, are whenever possible, consistent with usage in Holmes (1975). We will use the symbol 0 for both the zero element and subspace of a vector space.

\section{Facial reduction}

In her recent paper, Massam (1979) has generalized the BBZ conditions given for $Y=\mathbf{R}^{m}, S=\mathbf{R}_{+}^{m}$, see Abrams and Kerzner (1978), Ben-Israel and others (1976) and Ben-Tal and Ben-Israel (1979). She considered (P) as in (2.1) with $S$ closed pointed with interior, $\Omega=X$ a Banach space and $p, g$ Fréchet differentiable. This extension used the exposed faces of $S$ and was in fact flawed as we indicate below. More significantly no direct multiplier relationship was adduced. By studying the faces rather than exposed faces we are able to rectify both these situations. In this section we first introduce the necessary facial notions and 
prove a variety of preliminary results, see for example Barker (1973) and Barker and Carlson (1975). Most of the definitions and several of the results are modifications of those given by Massam, see Remarks 3.1-3.4.

Definition 3.1 (a) $K$ is a face of a convex cone $S$ if $K$ is a convex subset of $S$, and whenever

$$
s_{1} \text { and } s_{2} \text { lie in } S \text {, }
$$

then

$$
\frac{1}{2}\left(s_{1}+s_{2}\right) \in K \Rightarrow s_{1}, s_{2} \in K .
$$

(b) A face $K$ is exposed if it is the intersection of $S$ with the null space of a positive linear functional. That is: there exists $x^{\prime}$ in $S^{+}$with

$$
K=\left\{x \in S: x^{\prime} x=0\right\} \text {. }
$$

Such an $x^{\prime}$ is said to expose $K$ and of necessity is a supporting functional for $S$. Note that in our usage $S$ is itself exposed by the zero functional. Note also that faces of a convex cone $S$ are convex cones and are closed when $S$ is closed. These definitions of face and exposed face are equivalent to those in Massam (1979).

As is well known there are cones in which some faces are not exposed. We will have occasion to use the following example in the sequel.

EXAMPLE 3.1. Let $S_{1}$ denote the "ice-cream cone" in $\mathbf{R}^{3}$

$$
S_{1}=\left\{(x, y, z): 2 x y \geqslant z^{2}, x+y \geqslant 0\right\},
$$

and let $S_{2}$ denote

$$
S_{2}=\operatorname{coco}\left\{S_{1} \cup(1,0,1)\right\} .
$$

Then the nontrivial faces of $S_{1}$ are exactly the boundary rays and all the faces are exposed. In $S_{2}$, however, the ray through $(1,0,0)$ is extreme but not exposed and the smallest exposed face containing that ray is the cone generated by $(1,0,0)$ and $(1,0,1)$.

We will call a convex cone facially exposed if every face is exposed. Thus every polyhedral cone is facially exposed as is $S_{1}$.

Proposition 3.1. If $K$ is a face of $S$ then

$$
(K-K) \cap S=K \text {. }
$$

Proof. Suppose $s=k_{1}-k_{2}$ with $s$ in $S$ and $k_{1}, k_{2}$ in $K$. Then $s+k_{2}=k_{1}$ is in $K$ and by (3.1) $s$ lies in $K$. 
It is easy to see that the intersection of faces is a face. It is somewhat harder to see that the intersection of exposed faces is an exposed face.

Proposition 3.2. Let $C$ be an arbitrary subset of $S$. Then

(a) there is an unique minimal face $C^{f}$ containing $C$;

(b) there is an unique minimal exposed face $C^{\text {ef }}$ containing $C$.

Proof. (a) $C^{f}$ is just the intersection of all faces of $S$ containing $C$.

(b) The intersection of all exposed faces containing $C$ is a face containing $C$. We must show it is exposed.

Now since $S$ is finite dimensional the intersection of two non-nested faces has lower dimension than either face, Rockafellar (1970), Corollary 18.1.3. Hence the intersection of all exposed faces containing $C$ can in fact be replaced by the intersection of a finite subfamily $K_{i}, i=1,2, \ldots m$. Let $x_{i}^{\prime}$ expose $K_{i}$. Then $\sum_{i=1}^{m} x_{i}^{\prime}$ exposes $\cap_{i=1}^{m} K_{i}$ and this latter face is exposed and is the desired minimal face.

We now wish to identify faces of $S$ directly related to (P).

Definition 3.2. The minimal cone of $(P)$ denoted $S^{f}$, is defined by

$$
S^{f}=(-g(A))^{f}
$$

where $A$ is the feasible set of $(\mathrm{P})$. Similarly the minimal exposed cone for $(\mathrm{P})$ is denoted $S^{e f}$ and defined by

$$
S^{e f}=(-g(A))^{e f} .
$$

Note that $-g(A)$ lies in $S$. Proposition 3.2 guarantees that $S^{e f}$ and $S^{f}$ are well defined. $S^{e f}$ is essentially the cone $S^{=}$(or $B^{-}$) used by Massam (1979).

Proposition 3.3.

$$
S^{e f}=\frac{(g(A)+S)^{+\perp} \cap S .}{-\bigcup_{\lambda>0} \lambda(g(A)+S) \cap S .} .
$$

Proof. First, since $S$ is a cone and $g(A) \subset-S$, we have

$$
S^{+} \cap(g(A))^{\perp}=S^{+} \cap(g(A))^{+}=(S+g(A))^{+} .
$$

Now, since $K$ is an exposed face of $S$ exposed by $\phi$ and containing $-g(A)$ if and only if

$$
\phi \in S^{+} \cap(g(A))^{\perp},
$$


we get that

$$
\begin{aligned}
S^{e f} & =\cap\{\text { exposed faces of } S \text { containing }-g(A)\} \\
& =\left\{y \in S: \phi \in S^{+} \cap(g(A))^{\perp} \Rightarrow \phi y=0\right\} \\
& =S \cap\left\{y: \phi \in(S+g(A))^{+} \Rightarrow \phi y=0\right\}, \quad \text { by (3.10) } \\
& =S \cap(S+g(A))^{+\perp}
\end{aligned}
$$

which establishes (a). Now, by (2.10)

$$
S \cap(S+g(A))^{+\perp}=S \cap(S+g(A))^{++} \cap-(S+g(A))^{++} \text {. }
$$

Since $S$ lies in $(S+g(A))^{++}$this later set may be omitted. Finally since $g(A)+S$ is convex, (3.9) follows from (2.8).

Thus $S^{e f}$ has a pleasant overt description which needs no mention of faces.

Proposition 3.4. $S^{e f}$ and $S^{f}$ coincide for all (linear) constraint functions $g$ exactly when $S$ is facially exposed.

Proof. It is immediate that $S^{e f}$ and $S^{f}$ coincide if $S$ is facially exposed. Conversely suppose $K$ is a face of $S$ which is not exposed. Let $P$ be the orthogonal projection of $Y$ on $K-K$. In (2.1) set $g=P, \Omega=Y=X$. Then

$$
g(A)=P\left(P^{-1}(-S \cap(K-K))\right)=-S \cap(K-K)=-K,
$$

by Proposition (3.1). Thus $S^{f}=K$ while $S^{e f}$ must perforce contain $K$ and, being exposed, is not equal to $K$.

Example 3.2. We let $Y$ be the set of $m \times m$ real symmetric matrices and set $S$ to be the cone of positive semi-definite (psd) matrices in $Y$. The matrices are represented by their distinct triangular parts and thus $Y=\mathbf{R}^{\left(m^{2}+m\right) / 2}$. The norm on $Y$ is given by the Euclidean inner-product

$$
\langle A, B\rangle=\operatorname{tr} A B \text {, }
$$

the trace of the matrix product $A B$. The ice-cream cone $S_{1}$ in Example 3.1 represents the cone of $2 \times 2$ psd matrices in $\mathbf{R}^{3}$ and as we have seen is facially exposed. In fact, in general the cone $S$ of $m \times m$ psd matrices is self-dual, Berman and Ben-Israel (1969), and as we now prove, is facially exposed.

Proof. It was shown in Barker and Carlson (1975) that $K$ is a face of $S$ if and only if

$$
K=\{A \in S: \mathscr{T}(A) \supset \mathscr{R}(P)\},
$$

where $\mathscr{U}(A)$ denotes the null space of $A$ and $\mathcal{R}(P)$ is the range of a projection $P$ in $S$, that is $P^{2}=P$. To show that $K$ is an exposed face it is now sufficient to 
show that

$$
K=\{P\}^{\perp} \cap S
$$

since for any projection $P, \operatorname{tr} P A \geqslant 0$ for all psd $A$. Now, if $A \in K$, then $A \in S$ and $\Re(P) \subset \Re(A)$ implies that $\operatorname{tr} A P=\operatorname{tr} 0=0$ which implies that $A \in$ $\{P\}^{\perp}$. Thus $A \in\{P\}^{\perp} \cap S$. Conversely, suppose that $A \in\{P\}^{\perp} \cap S$. Then

$$
0=\operatorname{tr} A P=\operatorname{tr}(A P) P=\operatorname{tr} P(A P)=\sum_{i=1}^{m}\left(P e_{i}\right)^{t} A\left(P e_{i}\right)
$$

where $e_{i}$ are the unit vectors in $\mathbf{R}^{m}$ and ${ }^{t}$ denotes transpose. Since $A$ is psd, the above implies that $\left(P e_{i}\right)^{t} A\left(P e_{i}\right)=0$, for each $i$, which implies that $\mathscr{R}(P) \subset$ $\mathfrak{K}(A)$. Thus $A \in K$.

The following lemma exhibits the relationship between $S^{f}$-convexity of $g$ and convexity of the set $g(A)+S^{f}$.

LemMa 3.1. (a) the constraint $g$ is $S^{f}$-convex on the feasible set $A$.

(b) In particular, $g(A)+S^{f}$ is convex.

Proof. (a) Let $x_{1}, x_{2}$ lie in $A$ and $t$ lie in [0, 1]. Then

$$
g\left(t x_{1}+(1-t) x_{2}\right) \in g(A), \quad \operatorname{tg}\left(x_{1}\right)+(1-t) g\left(x_{2}\right) \in-S^{f}
$$

since $-S^{f}$ is convex and contains $g(A)$. Thus

$$
\operatorname{tg}\left(x_{1}\right)+(1-t) g\left(x_{2}\right)-g\left(t x_{1}+(1-t) x_{2}\right) \in S^{f}-S^{f} .
$$

Also as $g$ is $S$-convex, we get

$$
\operatorname{tg}\left(x_{1}\right)+(1-t) g\left(x_{2}\right)-g\left(t x_{1}+(1-t) x_{2}\right) \in S .
$$

By Proposition 3.1, (3.13) and (3.14) combine to show

$$
\operatorname{tg}\left(x_{1}\right)+(1-t) g\left(x_{2}\right)-g\left(t x_{1}+(1-t) x_{2}\right) \in S^{f}
$$

as desired. (b) now follows since $A$ is convex.

Recall that every convex set $C$ in finite dimensions has non-empty relative interior, denoted ri $C$, which is the interior of $C$ viewed as a subset of its affine span, Rockafellar (1970). The next lemma is fundamental to our subsequent results.

Lемма 3.2. If the feasible set $A$ is non-empty, then

$$
g(A) \cap-\text { ri } S^{f} \neq \varnothing .
$$


Proof. Suppose not, then

$$
\left(g(A)+S^{f}\right) \cap-\operatorname{ri} S^{f}=\varnothing
$$

since ri $S^{f}+S^{f} \subset$ ri $S^{f}$. Now considered as subsets of $S^{f}-S^{f}, g(A)+S^{f}$ and - ri $S^{f}$ are disjoint, non-empty convex sets and - ri $S^{f}$ is open. By the Separation Theorem there is a linear functional $y^{\prime}$ which may be supposed defined on $Y$ with

$$
y^{\prime}\left(g(A)+S^{f}\right) \leqslant 0, \quad y^{\prime}\left(- \text { ri } S^{f}\right)>0 .
$$

Since $S^{f}$ is a cone, the above implies that $y^{\prime}(g(A)) \leqslant 0$ and $y^{\prime}\left(S^{f}\right) \leqslant 0$. Therefore, since $g(A)$ lies in $-S^{f}$, it follows that $y^{\prime} g(A)=0$. Then $K=\{y \in Y$ : $\left.y^{\prime} y=0\right\} \cap S^{f}$ is a proper face of $S^{f}$ containing $g(A)$ which is impossible. Thus (3.15) must hold.

Remark 3.1. (i) Massam (1979), Lemma 4.3, wrongly claims (3.15) with $S^{f}$ replaced by $S^{e f}$. The error in the proof lies in the assumption that: if $H$ is a supporting hyperplane to $S^{\text {ef }}$, but not containing all of $S^{e f}$, then it can be assumed that $H$ is a supporting hyperplane to all of $S$. (In Example 3.1, the two dimensional cone $S_{3}=\operatorname{coco}\{(1,0,0) \cup(1,0,1)\}$ has a supporting hyperplane which contains the ray through $(1,0,0)$, but the only hyperplane which supports the cone $S_{2}$ and contains the ray through $(1,0,0)$ is the hyperplane which contains the cone $S_{3}$.) If, in fact, $g(A) \cap-$ ri $S^{e f}$ is non-empty, then the definition of $S^{f}$ implies that $S^{e f}$ and $S^{f}$ must coincide. By Proposition 3.4, this can only happen in generality if $S$ is facially exposed.

(ii) (3.15) shows that when $S$ has interior, then $S^{f}=S$ exactly when (P) has a Slater point.

We now present a corrected and simplified refinement of Theorem 4.2 in Massam (1979). Two subsidiary definitions are needed.

Definition 3.2. (a) Suppose that $\nabla g(a ; \cdot)$ exists. The generalized cone of constancy for (P) at $a$ is defined by

$$
\begin{gathered}
C^{f}(a)=\left\{d: \exists \alpha(d)>0, g(a+t d)-g(a)-\nabla g(a ; t d) \in S^{f}-S^{f},\right. \\
\left.g(a)+\nabla g(a ; t d) \in-\text { ri } S^{f}, \text { if } 0<t<\alpha(d)\right\}
\end{gathered}
$$

(b) $C^{e f}(a)$ is defined analogously, with $S^{e f}$ replacing $S^{f}$ throughout (3.18).

REMARK 3.2. If $g$ is supposed differentiable at $a$ and $S^{e f}-S^{e f}$ is reduced to $S^{\text {ef }}$ (which yields a formally smaller, though equal by (2.14) and (3.5), cone) $C^{e f}(a)$ coincides with Massam's definition, for $\Omega=X$.

The following theorem and corollary present representations of the above generalized cones of constancy in terms of feasible directions. 
Theorem 3.1. Let a lie in $A$.

(3.19) $\operatorname{cone}(\Omega-a) \cap C^{f}(a)=\operatorname{cone}(\Omega-a) \cap \operatorname{cone}\left(g^{-1}\left(-\operatorname{ri} S^{f}\right)-a\right)$;

(3.20) $\operatorname{cone}(\Omega-a) \cap C^{e f}(a)=\operatorname{cone}(\Omega-a) \cap \operatorname{cone}\left(g^{-1}\left(-\right.\right.$ ri $\left.\left.S^{e f}\right)-a\right)$;

ProOf. (a) Let us call the cone on the right of (3.19) $K^{f}$. Then suppose $d$ lies in $K^{f}$. For small positive $t, a+t d \in \Omega$ and $g(a+t d) \in-$ ri $S^{f}$. Thus both $a$ and $a+t d$ are in $A$. Since $g$ is $S^{f}$-convex on $A$ and since $S^{f}-S^{f}$ is closed, we get

$$
g(a+t d)-g(a)-\nabla g(a ; t d) \in \bar{S}^{f} \subset S^{f}-S^{f} .
$$

Thus as $g(a+t d)$ lies in - ri $S^{f}$ so does $g(a)+\nabla g(a ; t d)$, and $d$ lies in $C^{f}(a)$ and also in cone $(\Omega-a)$.

Conversely suppose $d$ lies in $C^{f}(a)$. Pick a neighbourhood $N$ of 0 with

$$
\nabla g(a ; t d)+g(a)+N \cap\left(S^{f}-S^{f}\right) \subset-\text { ri } S^{f}
$$

for $0<t<t_{1}$. Since for $t$ sufficiently small and positive

$$
g(a+t d)-g(a)-\nabla g(a ; t d) \in t N \subset N
$$

and also lies in $S^{f}-S^{f}$ by hypothesis, adding (3.23) and (3.22) yields $g(a+t d)$ $\in-$ ri $S^{f}$. This establishes (a). Part (b) is identical.

Corollary 3.1. (a) $\operatorname{cone}(\Omega-a) \cap C^{f}(a)$ is always non-empty but cone $(\Omega-a) \cap C^{e f}(a)$ is only non-empty if $S^{e f}=S^{f}$.

(b) $\overline{\operatorname{cone}(\Omega-a) \cap C^{f}(a)}=\overline{\operatorname{cone}}(A-a)$.

Proof. (a) Let $g(\hat{x})$ lie in $g(A) \cap-$ ri $S^{f}$ as promised by (3.15). Then $\hat{d}=\hat{x}-$ $a$ lies in cone $(\Omega-a) \cap C^{f}(a)$ by (3.19) and Lemma 3.1. The result now follows from (3.20) and Remark 3.1.

(b) Since $\Omega$ and $g^{-1}\left(-S^{f}\right)$ are convex,

$$
\operatorname{cone}(\Omega-a) \cap \operatorname{cone}\left(g^{-1}\left(-S^{f}\right)-a\right)=\operatorname{cone}(A-a) \text {. }
$$

It follows from (3.19) that

$$
\operatorname{cone}(\Omega-a) \cap C^{f}(a) \subset \operatorname{cone}(A-a) .
$$

Conversely, if $d$ lies in cone $(A-a)$ one has

$$
g(a+t d) \in-S^{f} ; \quad a+t d \in \Omega
$$

for $0<t<t_{1}$. If $\hat{d}=\hat{x}-a$ is as above it follows, as $g$ is $S^{f}$-convex on $A$, that for $0<\lambda<1$

$$
\begin{aligned}
g(a+t(\lambda \hat{d}+(1-\lambda) d)) & \in \lambda g(a+t \hat{d})+(1-\lambda) g(a+t d)-S^{f} \\
& \subset-\operatorname{ri} S^{f}-S^{f}-S^{f} \subset-\text { ri } S^{f}
\end{aligned}
$$


and $a+t(\lambda \hat{d}+(1-\lambda) d)$ lies in $\Omega$. Thus

$$
\lambda \hat{d}+(1-\lambda) d \in \operatorname{cone}(\Omega-a) \cap C^{f}(a) .
$$

Letting $\lambda$ go to zero we see that

$$
\operatorname{cone}(A-a) \subset \overline{\operatorname{cone}(\Omega-a) \cap C^{f}(a)} .
$$

Then (3.24) and (3.25) yield (b).

Remark 3.3. Massam (1979), Theorem 4.2 uses the set

$$
\text { cone }(\Omega-a) \cap C^{e f}(a)
$$

(with $\Omega$ set equal to $X$. See Remark 3.2). By virtue of part (a) of the above corollary, her result is true if and only if $S^{f}=S^{e f}$. In light of this we will now restrict our attention to $S^{f}$ and $C^{f}(a)$.

Theorem 3.2. Suppose that $a$ is feasible for $(P)$ and $p$ is continuous at $a$. Then the following are equivalent

$$
\text { a is optimal for }(P) \text {, }
$$

and

(3.27) (ii) $\quad\{d \in X: \nabla p(a ; d)<0\} \cap C^{f}(a) \cap \operatorname{cone}(\Omega-a)=\varnothing$.

Proof. It is standard that optimality for $(\mathrm{P})$ is characterized by

$$
\{d \in X: \nabla p(a ; d)<0\} \cap \operatorname{cone}(A-a)=\varnothing .
$$

Since $p$ is continuous at $a, \nabla p(a ; \cdot)$ is continuous and so (3.28) is equivalent to

$$
\{d \in X: \nabla p(a ; d)<0\} \cap \overline{\text { cone }}(A-a)=\varnothing .
$$

By (b) of Corollary 3.1 and the continuity of $\nabla p(a ; \cdot)$, this is equivalent to (3.27) as claimed.

RemarK 3.4. (i) Theorem 3.2 essentially presents a corrected and extended version of Theorem 4.2 of Massam (1979). Theorem 4.1 of Massam (1979) can in fact also be deduced from it. If $S$ has non-empty interior the exact statement of Massam's result is easily recovered (with $S^{f}$ replacing $S^{e f}$ of course).

(ii) If we apply the Dubovitskii-Milyutin Theorem, for example Holmes (1975), to the cones (which are convex)

and

$$
K=\{d \in X: \nabla p(a ; d)<0\}
$$

$$
G=\overline{C^{f}(a) \cap \operatorname{cone}(\Omega-a)},
$$


then Theorem 3.2 yields the dual characterization

$a$ (feasible) is optimal for (P) if and only if

$$
0 \in \partial p(a)-G^{+} \text {. }
$$

This is one part of Theorem 4.3 in Massam (1979) (with $S^{\text {ef }}$ replaced by $S^{f}$ ). The other part follows from the standard Lagrange multiplier theorem, since Slater's condition holds. We would like to get an analogue to the BBZ conditions even when Slater's condition fails. In Borwein and Wolkowicz (1980) we show that

$$
\mu=\inf \left\{p(x)+\lambda g(x): x \in \Omega \cap g^{-1}\left(S^{f}-S\right)\right\},
$$

for some $\lambda$ in $\left(S^{f}\right)^{+}$. In addition, if $\mu=p(a)$, for some $a$ in $A$, then

$$
\lambda g(a)=0
$$

and (3.32) and (3.33) characterize optimality of $a$ in $A$. This result directly yields the BBZ conditions if $S$ is $R_{+}^{m}$.

\section{Acknowledgement}

The authors would like to thank the referees for their many helpful comments.

\section{References}

R. A. Abrams, and L. Kerzner (1978), 'A simplified test for optimality', J. Optimization Theory Appl. 25, $161-170$.

G. P. Barker (1973), 'The lattice of faces of a finite dimensional cone', Linear Algebra and Appl. 7, 71-82.

G. Barker and D. Carlson (1975), 'Cones of diagonally dominant matrices', Pacific J. Math. 57, $15-32$.

A. Ben-Israel, A. Ben-Tal and S. Zlobec (1976), 'Optimality conditions in convex programming', The IX International Symposium of Mathematical Programming (Budapest, Hungary, August).

A. Ben-Tal and A. Ben-Israel (1979), 'Characterization of optimality in convex programming: the nondifferentiable case', Applicable Anal. 9, 137-156.

A. Berman and A. Ben-Israel (1969), 'Linear equations over cones with interior: a solvability theorem with applications to matrix theory', (Report No. 69-1, Series in Applied Math., Northwestern University).

J. Borwein (1978), 'Weak tangent cones and optimization in a Banach space', SIAM J. Control Optimization 16, 512-522.

J. Borwein (1980), 'Continuity and differentiability of convex operators', Proc. London Math. Soc., to appear.

J. M. Borwein and H. Wolkowicz (1979a), 'Regularizing the abstract convex program', J. Math. Anal. Appl., to appear.

J. Borwein and H. Wolkowicz (1979b), 'Characterizations of optimality without constraint qualification for the abstract convex program', (Research Report No. 14, Dalhousie University, Canada).

J. M. Borwein and H. Wolkowicz (1980), 'Characterization of optimality for the abstract convex program with finite dimensional range space', J. Austral. Math. Soc., to appear. 
B. D. Craven and S. Zlobec (1980), 'Complete characterization of optimality for convex programming in Banach spaces', Applicable Anal. 11, 61-78.

F. J. Gould and J. W. Tolle (1972), 'Geometry of optimality conditions and constraint qualifications', Math. Programming 2, 1-18.

R. B. Holmes (1975), Geometric functional analysis and its applications (Springer-Verlag).

H. Massam (1979), 'Optimality conditions for a cone-convex programming problem', J. Austral. Math. Soc. Ser. A. 27, 141-162.

A. L. Peressini (1967), Ordered topological vector spaces (Harper and Row).

A. P. and W. J. Robertson (1964), Topological vector spaces (Cambridge University Press).

R. T. Rockafellar (1970), Convex analysis (Princeton University Press).

J. Zowe (1974), 'Subdifferentiability of convex functions with values in an ordered vector space', Math Scand. 34, 69-83.

J. Zowe (1975), 'Linear maps majorized by a sublinear map', Arch. Math. (Basel), 26, 637-645.

Department of Mathematics

Dalhousie University

Halifax

Canada
Department of Mathematics

University of Alberta

Edmonton

Canada 